\title{
ON THE PEELING PROCEDURE APPLIED TO A POISSON POINT PROCESS
}

\author{
Y. DAVYDOV, ${ }^{*}$ Université de Lille 1 \\ A. NAGAEV, Nicolaus Copernicus University \\ A. PHILIPPE, ${ }^{* *}$ Université de Nantes
}

\begin{abstract}
In this paper we focus on the asymptotic properties of the sequence of convex hulls which arise as a result of a peeling procedure applied to the convex hull generated by a Poisson point process. Processes of the considered type are tightly connected with empirical point processes and stable random vectors. Results are given about the limit shape of the convex hulls in the case of a discrete spectral measure. We give some numerical experiments to illustrate the peeling procedure for a larger class of Poisson point processes.

Keywords: Control measure; convex hull; limiting shape; peeling; Poisson point process; stable vector

2010 Mathematics Subject Classification: Primary 60D05

Secondary 60G52; 60G55
\end{abstract}

\section{Introduction}

Consider a Poisson point process (PPP) $\pi=\pi_{\alpha, v}$ with points scattered over $\mathbb{R}^{d}$. Identify $\mathbb{R}^{d} \backslash\{\boldsymbol{0}\}$ with $\mathbb{R}_{+}^{1} \times S^{d-1}$, where $S^{d-1}$ is the unit sphere. Assume that the intensity measure of this process $\mu$ is of the form

$$
\mu=\theta \times \nu .
$$

Here $\theta$ is the absolutely continuous measure on $\mathbb{R}_{+}^{1}$ determined by the density function

$$
\frac{\mathrm{d} \theta}{\mathrm{d} \lambda}(r)=\alpha r^{-\alpha-1}, \quad r>0,
$$

$\lambda$ is the Lebesgue measure in $\mathbb{R}^{1}$, and $\alpha>0$ is a parameter. The spectral measure $v$ is a bounded measure on the $\sigma$-algebra $\mathcal{B}\left(S^{d-1}\right)$ of Borel subsets of $S^{d-1}$. Without loss of generality, we assume that $v\left(S^{d-1}\right)=1$. We denote by $S_{v}$ the support of $v$, and by cone $\left(S_{v}\right)$ the cone generated by $S_{v}$.

Representation (1) means that, for any Borel $A \subset \mathbb{R}^{1}$ and $E \subset S^{d-1}$,

$$
\mu\left\{x:|x| \in A, e_{x} \in E\right\}=\theta(A) v(E),
$$

where $e_{x}=|x|^{-1} x$ for all $x \in \mathbb{R}^{d}$.

\footnotetext{
Received 8 December 2008; revision received 1 March 2010.

The main results of this paper were obtained together with Alexander Nagaev, with whom the first author had collaborated for more than 35 years, until Alexander's tragic death in 2005. Since then, we have gathered strength and finalised this paper, strongly feeling Alexander's absence-our memories of him will stay with us forever.

* Postal address: Laboratoire Paul Painlevé, Université de Lille 1 Batiment M2, 59655 Villeneuve d'Ascq Cedex, France.

** Postal address: Laboratoire de Mathématiques Jean Leray, Université de Nantes, 2 rue de la Houssinière, 44322 Nantes Cedex 3, France. Email address: anne.philippe@univ-nantes.fr
} 
Let $B(\mathbf{0}, r)$ denote the ball of radius $r$ centered at the origin. It is easily seen that, for any $\delta>0, \mu(B(\mathbf{0}, \delta))=\infty$ and $\mu\left(\mathbb{R}^{d} \backslash B(\mathbf{0}, \delta)\right)<\infty$, that is, with probability 1 , in any neighborhood of the origin there are infinitely many points of $\pi$ while $\pi\left(\mathbb{R}^{d} \backslash B(\mathbf{0}, \delta)\right)$ is finite.

Our interest in the point processes controlled by (1) is explained by the following facts.

Let $\xi^{(1)}, \ldots, \xi^{(n)}$ be independent copies of a random vector $\xi$ with the distribution $\mathrm{P}_{\xi}$ such that the function $\mathrm{P}\{|\xi|>r\}$ regularly varies as $r \rightarrow \infty$ with exponent $-\alpha$ and the measures $\left(v_{r}\right)_{r}$ defined by

$$
v_{r}(E)=\mathrm{P}\left\{e_{\xi} \in E:|\xi|>r\right\}, \quad E \in \mathcal{B}\left(S^{d-1}\right),
$$

weakly converging to $v$ on $B^{\mathrm{c}}(\mathbf{0}, \tau)$ for any $\tau>0$.

Consider the empirical point process $\beta_{n}$ generated by $\xi^{(1)}, \ldots, \xi^{(n)}$ or, more precisely, by the random set

$$
\varsigma_{1}^{(n)}=\left\{b_{n}^{-1} \xi^{(1)}, \ldots, b_{n}^{-1} \xi^{(n)}\right\}=\left\{\hat{\xi}^{(1)}, \ldots, \hat{\xi}^{(n)}\right\},
$$

where

$$
b_{n}=\inf \left\{r: n \mathrm{P}\left\{e_{\xi} \in E:|\xi|>r\right\} \leq 1\right\} .
$$

It is easily seen that the point process $\beta_{n}$ weakly converges to $\pi=\pi_{\alpha, v}$; see, e.g. [4, Proposition 3.21]. Thus, each $\pi_{\alpha, v}$ is a weak limit of a sequence of empirical processes.

It can be easily established that $\pi_{\alpha, v}$ admits the following representation:

$$
\pi_{\alpha, v}=\sum_{k=1}^{\infty} \delta_{\left\{\Gamma_{k}^{-1 / \alpha} \epsilon_{k}\right\}},
$$

where $\Gamma_{k}=\sum_{i=1}^{k} \gamma_{k}$ for the sequence $\left(\gamma_{k}\right)_{k \in \mathbb{N}}$ of independent, identically exponentially distributed random variables with mean 1 , and $\left(\epsilon_{k}\right)_{k \in \mathbb{N}}$ is a sequence of independent and identically distributed (i.i.d.) random elements in $S^{d-1}$ with common distribution $v$, independent of $\left(\gamma_{k}\right)_{k \in \mathbb{N}}$; see, e.g. [2]. It is worth recalling that the point processes $\pi_{\alpha, v}$ naturally arise within the framework of the theory of stable distributions. For example, if $\alpha \in(0,1)$ then the series $\zeta=\sum_{x(j) \in \pi_{\alpha, v}} x^{(j)}$ converges almost surely (a.s.) and has the $d$-dimensional stable distribution; see, e.g. [2], [3], and [5, Chapter 2].

Definition 1. We say that the underlying distribution $\mathrm{P}_{\xi}$ and the corresponding spectral measure $v$ (as well as the point process $\pi_{\alpha, v}$ ) are nonunilateral if $v$ is supported by a set $S_{v} \subset S^{d-1}$ such that cone $\left(S_{v}\right)=\mathbb{R}^{d}$.

In this paper we focus on the sequence of convex hulls that arise from the peeling procedure introduced in [1]. In what follows, $C(A)$ denotes the convex hull generated by $A \subset \mathbb{R}^{d}$ and ext $C$ denotes the set of extreme points of a convex set $C$. If $C$ is a convex polyhedron then ext $C$ is the finite set of its vertices.

It is convenient to start with the binomial process $\left(\varsigma_{1}^{(n)}\right)$. Let $C_{1}^{(n)}=C\left(\varsigma_{1}^{(n)}\right)$. If the measure $\mathrm{P}_{\xi}$, the distribution of $\xi$, is nonunilateral then, a.s., $C_{1}^{(n)}$ is a polyhedron and, furthermore,

$$
\varsigma_{1}^{(n)} \cap \partial C_{1}^{(n)}=\operatorname{ext} C_{1}^{(n)} \text {. }
$$

Define

$$
\varsigma_{2}^{(n)}=\varsigma_{1}^{(n)} \backslash \operatorname{ext} C_{1}^{(n)}, \quad C_{2}^{(n)}=C\left(\varsigma_{2}^{(n)}\right)
$$

then

$$
\varsigma_{3}^{(n)}=\varsigma_{2}^{(n)} \backslash \operatorname{ext} C_{2}^{(n)}, \quad C_{3}^{(n)}=C\left(\varsigma_{3}^{(n)}\right),
$$

and so on. Obviously, the sequence of these nonempty convex hulls, $\left(C_{k}^{(n)}\right)_{k \in \mathbb{N}}$, is finite and its length is random. 
If $0<\alpha<2$ and $v$ is nonunilateral, then $\left(C_{0}^{(n)}, C_{1}^{(n)}, \ldots, C_{k}^{(n)}\right)$ as $n \rightarrow \infty$ converges in distribution to $\left(C_{0}, C_{1}, \ldots, C_{k}\right)$ for any fixed $k$; see [1]. Consequently, the sequence\#\{ext $\left.C_{k}^{(n)}\right\}$ is bounded in probability as $n$ tends to $\infty$. Here \#A denotes the cardinality of the set $A$.

In order to learn how $C_{k}^{(n)}$ relates to $C_{k}$ when $k=k_{n} \rightarrow \infty$, we need, first, to learn how $C_{k}$ behaves as $k \rightarrow \infty$. It should be noted that $C_{k}^{(n)}$ can be regarded as the multidimensional analog of the order statistics. So, the asymptotic properties of $C_{k_{n}}^{(n)}$ are of great interest from the viewpoint of mathematical statistics.

We now generalise the construction of the peeling sequence to an infinite set of points, $\varsigma=\varsigma_{1}$, the support of the random measure $\pi_{\alpha, \nu}$. We may apply to $\varsigma$ the same peeling procedure as in the case of the finite set $\varsigma_{1}^{(n)}$. As a result, we obtain the sequence of sets $\varsigma_{1}, \varsigma_{2}, \ldots$, the sequence of their convex hulls $C_{1}, C_{2}, \ldots$, and the sets of extreme points ext $C_{k}, k \geq 1$. Furthermore, $\varsigma_{k+1}=\varsigma_{k} \backslash \operatorname{ext} C_{k}$ a.s. If $v$ is nonunilateral then $\mathbf{0} \in C_{k}$ a.s., $C_{k}$ is a.s. a polyhedron, and $\varsigma_{k} \cap \partial C_{k}=\operatorname{ext} C_{k}$ for all $k$.

Intuitively, we expect that the asymptotic behavior of $C_{n}$ is rather regular. It is convenient to state our basic conjecture in the following way. Define

$$
\hat{C}_{n}=\rho_{n}^{-1} C_{n}, \quad \text { where } \rho_{n}=\max _{x \in C_{n}}|x| .
$$

Conjecture 1. If $v$ is nonunilateral then there exists a nonrandom set $\hat{C}$ such that

$$
\lim _{n \rightarrow \infty} d_{H}\left(\hat{C}_{n}, \hat{C}\right)=0 \text { a.s. }
$$

The set $\hat{C}$ (if it exists) is called the limit shape of the sequence $\hat{C}_{n}$. It is easy to show that if such a $\hat{C}$ exists then it is certainly nonrandom. Indeed, labeling the points of $\zeta$ in the descending order of their distances from the origin we obtain a sequence $x^{(1)}, x^{(2)}, x^{(3)}, \ldots$ such that, a.s.,

$$
\left|x^{(1)}\right|>\left|x^{(2)}\right|>\left|x^{(3)}\right|>\cdots \text {. }
$$

It is worth noting that the joint distribution of $\left|x^{(1)}\right|,\left|x^{(2)}\right|, \ldots,\left|x^{(n)}\right|$ is absolutely continuous with a density of the form

$$
p_{n}\left(r_{1}, r_{2}, \ldots, r_{n}\right)=\alpha^{n}\left(r_{1} r_{2} \cdots r_{n}\right)^{-\alpha-1} \exp \left(-v\left(S^{d-1}\right) r_{1}^{-\alpha}\right) \mathbf{1}_{\left\{r_{1}>r_{2}>\cdots>r_{n}\right\}} .
$$

Let $(\eta, \varepsilon),\left(\eta_{1}, \varepsilon^{(1)}\right),\left(\eta_{2}, \varepsilon^{(2)}\right), \ldots$ be i.i.d. with common distribution

$$
\mathrm{P}\{\eta>r, \varepsilon \in E\}=\mathrm{e}^{-r} \nu(E) .
$$

According to (2), we have

$$
\left\{x^{(j)}\right\}_{j=1}^{\infty} \stackrel{\mathrm{D}}{=}\left\{\varepsilon^{(j)}\left(\eta_{1}+\cdots+\eta_{j}\right)^{1 / \alpha}\right\}_{j=1}^{\infty},
$$

which implies that the event $\left\{\lim _{n \rightarrow \infty} \hat{C}_{n}\right.$ exists $\}$ belongs to the $\sigma$-algebra $\ell$ of the events invariant with respect to all finite permutations of the random vectors $\left\{\left(\eta_{k}, \varepsilon^{(k)}\right), k \in \mathbb{N}\right\}$. Here, 'D, denotes equality in distribution. By the Hewitt-Savage zero-one law, $l$ is trivial. Since the limit set $\hat{C}=\lim _{n \rightarrow \infty} \hat{C}_{n}$ is $\ell$-measurable, we conclude that $\hat{C}$ is constant with probability 1 .

Now we give a first example where the existence of the limit shape is proved.

Example 1. Let $S_{\nu}$ consist of $d+1$ unit vectors $e^{(1)}, \ldots, e^{(d+1)}$ such that cone $\left(e^{(1)}, \ldots, e^{(d+1)}\right)$ coincides with $\mathbb{R}^{d}$. Then $\pi_{\alpha, v}$ is decomposed on $d+1$ one-dimensional independent PPPs of the form $\left(x_{k}^{(i)}=\left|x_{k}^{(i)}\right| e^{(i)}\right)$. Since $v$ is nonunilateral, the points $x_{k}^{(i)}, i=1,2, \ldots, d+1$, serve as vertices of $C_{k}, k=1,2, \ldots$ Moreover, $\left|x_{k}^{(i)}\right| \stackrel{\mathrm{D}}{=}\left(v_{i}\right)^{1 / \alpha}\left(\eta_{1}+\cdots+\eta_{k}\right)^{1 / \alpha}$ and $\rho_{n} n^{1 / \alpha} \rightarrow t^{+}=\max _{1 \leq i \leq d+1}\left(v_{i}\right)^{1 / \alpha}$ a.s. Then the limit shape $\hat{C}$ is the convex polyhedron with vertices $v^{(i)}=\left(t_{i} / t^{+}\right) e^{(i)}$ and $t_{i}=\left(v_{i}\right)^{1 / \alpha}, i=1,2, \ldots, d+1$. 
Theorems 1 and 2, stated and proved below, deal with the case where a nonunilateral $v$ is supported by a finite number of unit vectors. If $\#\left(S_{v}\right)>d+1$ then the situation becomes much more complicated.

Intuitively, we expect that, say, in the case of $v$ uniformly distributed over $S^{d-1}$ the unit ball arises as the limit shape. However, it is not easy to prove this formally. The authors tried to verify the credibility of this conjecture using the Monte Carlo simulation. Obviously, representation (2) provides a basis for such a simulation. The results of the simulation presented in Section 4 make this conjecture very credible.

\section{Almost-sure convergence of the peeling}

In this section we assume that the spectral measure $v$ of the process $\pi_{\alpha, v}$ is atomic, i.e. it is supported by a finite number of the points $e^{(1)}, \ldots, e^{(l)}$ belonging to the unit sphere $S^{d-1}$. Furthermore, it is also assumed that cone $\left\{e^{(1)}, \ldots, e^{(l)}\right\}=\mathbb{R}^{d}$. Denote by $v_{i}=v\left(\left\{e^{(i)}\right\}\right), i=$ $1,2, \ldots, l$, the atoms of $v$.

Then the considered point process $\pi_{\alpha, v}$ is a superposition of the one-dimensional independent PPPs defined on the rays

$$
\mathcal{L}_{i}=\left\{x: x=t e^{(i)}, t>0\right\}, \quad i=1, \ldots, l,
$$

with intensities

$$
\mu_{i}(A)=v_{i} \alpha \int_{A} r^{-\alpha-1} \mathrm{~d} r, \quad A \in \mathcal{B}\left(\mathcal{L}_{i}\right) .
$$

Definition 2. Let $A=\left\{a^{(1)}, \ldots, a^{(m)}\right\} \subset \mathbb{R}^{q}$ be a finite set with $m \geq d+1$. The set $A$ is extreme if ext $C(A)=A$.

Theorem 1. Let $C_{k}\left(\pi_{\alpha, v}\right)$ be the kth convex hull of the PPP $\pi_{\alpha, v}$. Denote by $C_{\infty}$ the convex hull generated by $A=\left\{v_{1}^{1 / \alpha} e^{(1)}, \ldots, v_{l}^{1 / \alpha} e^{(l)}\right\}$. If $A$ is extreme then

$$
d_{H}\left(k^{1 / \alpha} C_{k}\left(\pi_{\alpha, \nu}\right), C_{\infty}\right)=O\left(\sqrt{\frac{1 \ln k}{k}}\right) \text { a.s. as } k \rightarrow \infty,
$$

where $\ln k=\ln \ln k$. The polyhedron $C_{\infty}$ determines the limit shape of the convex hulls $C_{k}\left(\pi_{\alpha, v}\right)$.

Remark 1. If $\sigma$ is uniformly distributed over its support in the sense that $v_{i}=l^{-1}$, then the total number of vertices of $C_{\infty}$ equals $l$. Furthermore, the vertices lie on the sphere of radius $l^{-1 / \alpha}$. Loosely speaking, the convex hulls $C_{k}\left(\pi_{\alpha, v}\right)$ become round as $k \rightarrow \infty$.

If the condition that $A$ is extreme is omitted, we can state the following result.

Theorem 2. Let $C_{k}\left(\pi_{\alpha, v}\right)$ be the kth convex hull of $\pi_{\alpha, v}$. Denote by $C_{\infty}$ the convex hull generated by $A=\left\{v_{1}^{1 / \alpha} e^{(1)}, \ldots, v_{l}^{1 / \alpha} e^{(l)}\right\}$. Then

$$
d_{H}\left(k^{1 / \alpha} C_{k}\left(\pi_{\alpha, v}\right), C_{\infty}\right) \rightarrow 0 \quad \text { a.s. as } k \rightarrow \infty .
$$

Remark 2. Let $f$ be a continuous homogeneous functional of degree $\gamma$ defined on convex sets. Theorem 2 implies that

$$
k^{\gamma / \alpha} f\left(C_{k}\left(\pi_{\alpha, \nu}\right)\right) \rightarrow f\left(C_{\infty}\right) \text { a.s. }
$$




\section{Proofs}

\subsection{Auxiliary lemmas}

Let $\eta_{1}, \eta_{2}, \ldots$ be i.i.d. random variables with the standard exponential distribution, so that $a=\mathrm{E} \eta_{1}=\operatorname{var} \eta_{1}=1$. Define the sum

$$
\Gamma_{n}=\eta_{1}+\cdots+\eta_{n}
$$

By the law of the iterated logarithm there exists an almost-sure finite random variable $\kappa$ with values in $\mathbb{N}$ such that, for $n \geq \kappa$,

$$
\left|n^{-1} \Gamma_{n}-1\right|<2 \sqrt{\frac{\ln n}{n}} \text { a.s. }
$$

Consider a function $h(z)=z^{-1 / \alpha}$. If $|z-1| \leq \frac{1}{2}$ then $|h(z)-h(1)| \leq L_{\alpha}|z-1|, L_{\alpha}<\infty$.

Let $n^{\prime}=\min \left\{n: 2 \sqrt{\ln n / n}<\frac{1}{2}\right\}$. If $n \geq \max \left(n^{\prime}, \kappa\right)$ then

$$
\left|h\left(n^{-1} \Gamma_{n}\right)-h(1)\right| \leq 2 L_{\alpha} \sqrt{\frac{\ln n}{n}},
$$

and, therefore, for $n \geq \kappa=\kappa(\omega)$,

$$
\left|\Gamma_{n}^{-1 / \alpha}-n^{-1 / \alpha}\right| \leq 2 L_{\alpha} \frac{\sqrt{\ln n}}{n^{1 / \alpha+1 / 2}} \quad \text { a.s. }
$$

We call any countable set of points from $\mathbb{R}^{d}$ such that, for any $\delta>0$, there are a finite number of points belonging to the set that lie outside the ball $\{x:|x| \leq \delta\}$ the configuration. So the point $\mathbf{0}$ is the limit point of any configuration.

We call a configuration $\varsigma$ nonunilateral if all convex hulls, $C_{k}=C_{k}(\varsigma), k=1,2, \ldots$, generated by $\varsigma$ contain $\mathbf{0}$ as an interior point. It is evident that, under the conditions of Theorem 1 , the random measure $\pi_{\alpha, v}$ is a.s. supported by a nonunilateral configuration $\varsigma$.

Denote by $\operatorname{int}(\varsigma)$ the set of the interior points of $\varsigma$, i.e. $\operatorname{int}(\varsigma)=\left\{x: x \in \varsigma, x \notin \partial C_{1}(\varsigma)\right\}$. Let $\mathcal{K}$ be the set of nonunilateral configurations such that no $d+1$ points lie on the same hyperplane.

Lemma 1. If $\varsigma_{1}, \varsigma_{2} \in \mathcal{K}$ and $\varsigma_{1} \subset \varsigma_{2}$, then, for all $k \in \mathbb{N}, C_{k}\left(\varsigma_{1}\right) \subset C_{k}\left(\varsigma_{2}\right)$.

Proof. It is trivial to prove that $C_{1}\left(\varsigma_{1}\right) \subset C_{1}\left(\varsigma_{2}\right)$. If $x$ is an interior point of $C_{1}\left(\varsigma_{1}\right)$, i.e. $x \in$ $\operatorname{int}\left(C_{1}\left(\varsigma_{1}\right)\right)$, then $x$ is also an interior point of $C_{1}\left(\varsigma_{2}\right)$; therefore, $\operatorname{int}\left(C_{1}\left(\varsigma_{1}\right)\right) \subset \operatorname{int}\left(C_{1}\left(\varsigma_{2}\right)\right)$, and this implies that

$$
C_{2}\left(\varsigma_{1}\right)=C_{1}\left(\operatorname{int}\left(C_{1}\left(\varsigma_{1}\right)\right)\right) \subset C_{1}\left(\operatorname{int}\left(C_{1}\left(\varsigma_{2}\right)\right)\right)=C_{2}\left(\varsigma_{2}\right) .
$$

By induction, the lemma is proved.

Lemma 2. Let $\varsigma, \varsigma^{\prime} \in \mathcal{K}$ be such that $\varsigma^{\prime} \subset \varsigma$ and $\#\left(\varsigma^{\prime} \backslash \varsigma\right)=m<\infty$. Then

$$
C_{k+m}(\varsigma) \subset C_{k}\left(\varsigma^{\prime}\right) \subset C_{k}(\varsigma), \quad k \in \mathbb{N} .
$$

Proof. Since $\varsigma, \varsigma^{\prime} \in \mathcal{K}$ and $\mathbf{0}$ is the only limit point of both configurations, all the $C_{k}(\varsigma), k=1,2, \ldots$, are polyhedrons. Note that, for all $k, l \geq 1$,

$$
C_{k+1}(\varsigma)=C_{1}\left(\operatorname{int}\left(C_{k}(\varsigma) \cap \varsigma\right)\right)
$$


and

$$
C_{k+l}(\varsigma)=C_{k}\left(\operatorname{int}\left(C_{l}(\varsigma) \cap \varsigma\right)\right) .
$$

First, let $m=1$. Note that the inclusion $C_{1}\left(\varsigma^{\prime}\right) \subset C_{1}(\varsigma)$ follows directly from the relation $\varsigma^{\prime} \subset \varsigma$. Define $\{a\}=\varsigma \backslash \varsigma^{\prime}$. Consider two possible cases, $a \notin C_{1}\left(\varsigma^{\prime}\right)$ and $a \in C_{1}\left(\varsigma^{\prime}\right)$.

Let $a \notin C_{1}\left(\varsigma^{\prime}\right)$. In this case $a \in \partial C_{1}(\varsigma)$, i.e. $C_{1}\left(\varsigma^{\prime}\right) \neq C_{1}(\varsigma)$. This implies that $\operatorname{int}(\varsigma) \subset \varsigma^{\prime}$. Utilising (9) with $k=1$ yields $C_{2}\left(\varsigma^{\prime}\right) \subset C_{1}(\varsigma)$. Since the inclusion $C_{1}\left(\varsigma^{\prime}\right) \subset C_{1}(\varsigma)$ is obvious, we conclude that (8) holds for $k=m=1$.

Assume that (8) holds for $m=1$ and all $k \leq n$, and show that it also holds for $m=1$ and $k=n+1$. By the induction assumption we have

$$
C_{n+1}(\varsigma) \subset C_{n}\left(\varsigma^{\prime}\right) \subset C_{n}(\varsigma)
$$

Since $\operatorname{int}\left(C_{n}\left(\varsigma^{\prime}\right) \cap \varsigma^{\prime}\right)=\operatorname{int}\left(C_{n}\left(\varsigma^{\prime}\right) \cap \varsigma\right)$, we obtain, taking into account (9),

$$
C_{n+l}\left(\varsigma^{\prime}\right)=C_{1}\left(\operatorname{int}\left(C_{n}\left(\varsigma^{\prime}\right) \cap \varsigma\right)\right) .
$$

From the right-hand side inclusion of (11), it follows that $C_{n+1}\left(\varsigma^{\prime}\right) \subset C_{n+1}(\varsigma)$. Furthermore, from the left-hand side inclusion of (11) we conclude that

$$
\operatorname{int}\left(C_{n+1}(\varsigma) \cap \varsigma\right)=\operatorname{int}\left(C_{n}\left(\varsigma^{\prime}\right) \cap \varsigma^{\prime}\right) .
$$

Applying (10) yields $C_{n+2}(\varsigma) \subset C_{n+1}\left(\varsigma^{\prime}\right)$. Thus, (8) holds for $k=n+1$ and $m=1$, i.e. the case $a \notin C_{1}\left(\varsigma^{\prime}\right)$ is completed.

If $a \in C_{1}\left(\varsigma^{\prime}\right)$ then there exists an integer $n_{0}$ such that

$$
C_{n}\left(\varsigma^{\prime}\right)=C_{n}\left(\varsigma^{\prime}\right), \quad n=1,2, \ldots, n_{0}, \quad C_{n_{0}+1}\left(\varsigma^{\prime}\right) \neq C_{n_{0}+1}\left(\varsigma^{\prime}\right) .
$$

Furthermore, $a \notin C_{n_{0}+1}\left(\varsigma^{\prime}\right)$. Obviously, (8) is trivial for $m=1$ and $n=1,2, \ldots, n_{0}$. Hence, it remains to apply the above argument to the configurations $C_{n_{0}+1}(\varsigma) \cap \varsigma$ and $C_{n_{0}+1}\left(\varsigma^{\prime}\right) \cap \varsigma^{\prime}$. Thus, the lemma is proved for all $k$ and $m=1$.

Now, let $m>1$, i.e. $\varsigma \backslash \varsigma^{\prime}=\left\{a_{1}, \ldots, a_{m}\right\}$. Consider the configurations

$$
\varsigma_{0}=\varsigma, \quad \varsigma_{1}=\varsigma \backslash\left\{a_{1}\right\}, \quad \varsigma_{2}=\varsigma \backslash\left\{a_{1}, a_{2}\right\}, \ldots, \quad \varsigma_{m}=\varsigma \backslash\left\{a_{1}, \ldots, a_{m}\right\}=\varsigma^{\prime} .
$$

Note that the neighboring configurations differ by a single point. So, we may apply (11). Applying (11) yields

$$
C_{k+m}(\varsigma) \subset C_{k+m-1}\left(\varsigma_{1}\right) \subset C_{k+m-2}\left(\varsigma_{2}\right) \subset \cdots \subset C_{k}\left(\varsigma_{m}\right)=C_{k}\left(\varsigma^{\prime}\right) \subset C_{k}(\varsigma) .
$$

This completes the proof.

\subsection{Proof of Theorem 1}

Lemma 3. Let $e^{(i)}$ for $i=1, \ldots, l$ with $l \geq d+1$ be unit vectors such that

$$
\text { cone }\left\{e^{(1)}, \ldots, e^{(l)}\right\}=\mathbb{R}^{d} .
$$

If $A=\left\{v_{1}^{1 / \alpha} e^{(1)}, \ldots, v_{l}^{1 / \alpha} e^{(l)}\right\}$ is extreme then there exist $r>0$ and $\varepsilon$ depending only on $A$ and on the dimensiond such that the set $\left\{r_{1} v_{1}^{1 / \alpha} e^{(1)}, \ldots, r_{l} v_{l}^{1 / \alpha} e^{(l)}\right\}$ is extreme for all $\left(r_{1}, \ldots, r_{n}\right)$ such that $\left|r_{i} / r-1\right|<\varepsilon, i=1, \ldots, l$.

Proof. The proof of this lemma is evident. 
Proof of Theorem 1. Let us label the points $x_{1}^{(i)}, x_{2}^{(i)}, \ldots$ lying on the ray $\mathcal{L}_{i}$ in descending order of their norms. Obviously, the sequences $\left\{x_{n}^{(i)}, n \in \mathbb{N}\right\}, 1 \leq i \leq l$, are jointly independent. By (4),

$$
\left\{\left|x_{n}^{(i)}\right|\right\} \stackrel{\mathrm{D}}{=}\left\{v_{i}^{1 / \alpha} \Gamma_{n}^{-1 / \alpha}\right\}
$$

where $\Gamma_{n}$ is defined as in (6).

Let $\epsilon>0$. According to (7), there exists $n_{0}=n_{0}(\omega)$ such that, for all $i=1, \ldots, l$ and all $n \geq n_{0}$,

$$
\left|x_{n}^{(i)}-v_{i}^{1 / \alpha} n^{-1 / \alpha}\right| \leq 2 L_{\alpha} n^{-1 / \alpha-1 / 2} \sqrt{\ln n}
$$

and

$$
2 L_{\alpha} n^{-1 / 2} \sqrt{\ln n}<\varepsilon .
$$

Let the configuration $\varsigma^{\prime}$ be formed by the points $x_{n}^{(i)}, n \geq n_{0}, i=1, \ldots, l$, i.e.

$$
\varsigma^{\prime}=\bigcup_{i=1}^{l}\left\{x_{n_{0}}^{(i)}, x_{n_{0}+1}^{(i)}, \ldots\right\} .
$$

Consider, for all $k \leq 1$,

$$
A_{k}^{+}=\left\{\left(n_{0}+k-1\right)^{-1 / \alpha}\left(1+\varepsilon_{k}\right) v_{1}^{1 / \alpha} e^{(1)}, \ldots,\left(n_{0}+k-1\right)^{-1 / \alpha}\left(1+\varepsilon_{k}\right) v_{l}^{1 / \alpha} e^{(l)}\right\}
$$

and

$$
A_{k}^{-}=\left\{\left(n_{0}+k-1\right)^{-1 / \alpha}\left(1-\varepsilon_{k}\right) v_{1}^{1 / \alpha} e^{(1)}, \ldots,\left(n_{0}+k-1\right)^{-1 / \alpha}\left(1-\varepsilon_{k}\right) v_{l}^{1 / \alpha} e^{(l)}\right\},
$$

where

$$
\varepsilon_{k}=2 L_{\alpha} \sqrt{\frac{\ln \left(k+n_{0}-1\right)}{k+n_{0}-1}} .
$$

By virtue of (12), the points $x_{n_{0}}^{(1)}, \ldots, x_{n_{0}}^{(l)}$ hit the layer $C\left(A_{1}^{+}\right) \backslash C\left(A_{1}^{-}\right)$. By Lemma 3 , the convex hull $C_{1}\left(\varsigma^{\prime}\right)$ is the polyhedron and

$$
\operatorname{ext} C_{1}\left(\varsigma^{\prime}\right)=\left\{x_{n_{0}}^{(1)}, \ldots, x_{n_{0}}^{(l)}\right\} .
$$

Similarly,

$$
\left\{x_{n_{0}+1}^{(1)}, \ldots, x_{n_{0}+1}^{(l)}\right\} \subset C\left(A_{2}^{+}\right) \backslash C\left(A_{2}^{-}\right),
$$

and, therefore, it is an extreme set, i.e.

$$
\operatorname{ext} C\left(\left\{x_{n_{0}+1}^{(1)}, \ldots, x_{n_{0}+1}^{(l)}\right\}\right)=\left\{x_{n_{0}+1}^{(1)}, \ldots, x_{n_{0}+1}^{(l)}\right\} .
$$

It is evident that

$$
\operatorname{ext} C_{2}\left(\varsigma^{\prime}\right)=\left\{x_{n_{0}+1}^{(1)}, \ldots, x_{n_{0}+1}^{(l)}\right\} .
$$

Continuing in this way we obtain the $k$ th convex hull $C_{k}\left(\varsigma^{\prime}\right)$ such that

$$
\operatorname{ext} C_{k}\left(\varsigma^{\prime}\right)=\left\{x_{n_{0}+k-1}^{(1)}, \ldots, x_{n_{0}+k-1}^{(l)}\right\} \subset C\left(A_{k}^{+}\right) \backslash C\left(A_{k}^{-}\right) .
$$

The last inclusion implies that

$$
d_{H}\left(\left(k+n_{0}-1\right)^{1 / \alpha} C_{k}\left(\varsigma^{\prime}\right), C_{\infty}\right) \leq \varepsilon_{k},
$$


where $C_{\infty}$ is the convex hull generated by $A=\left\{v_{1}^{1 / \alpha} e^{(1)}, \ldots, v_{l}^{1 / \alpha} e^{(l)}\right\}$. From (8), it follows that

$$
C_{k+m}\left(\varsigma^{\prime}\right) \subset C_{k+m}\left(\pi_{\alpha, v}\right) \subset C_{k}\left(\varsigma^{\prime}\right) \quad \text { with } m=\left(n_{0}-1\right) l
$$

Therefore,

$$
\text { ext } C_{k+m}\left(\pi_{\alpha, v}\right) \subset C\left(A_{k}^{+}\right) \backslash C\left(A_{k+m^{\prime}}^{-}\right) \quad \text { with } m^{\prime}=\left(n_{0}-1\right)(l-1) .
$$

So, for all sufficiently large $k$,

$$
d_{H}\left((k+m)^{1 / \alpha} C_{k+m}\left(\pi_{\alpha, v}\right), C_{\infty}\right) \leq 2 \varepsilon_{k} .
$$

Since $m$ is fixed, the theorem follows.

\subsection{Proof of Theorem 2}

Let $\epsilon$ be an arbitrary positive real. Hereafter, we denote by $A^{(\epsilon)}$ the $\epsilon$-neighborhood of a set $A$ :

$$
A^{(\epsilon)}=\{x: d(x, A)<\epsilon\} .
$$

The set $A_{1}=A \cap \partial C(A)=\left\{v_{j}^{1 / \alpha} e^{(j)}, j \in J\right\}$ is extreme. From the process $\pi_{\alpha, \nu}$, we construct a new PPP $\pi_{1}$ obtained by deleting all points on the rays $\mathcal{L}_{j}=\left\{x \mid x=t e^{(i)}, t>0\right\}$, $j \in J$. By Lemma 1 we have, for all $n \in \mathbb{N}$,

$$
C_{n}\left(\pi_{1}\right) \subset C_{n}\left(\pi_{\alpha, v}\right)
$$

Since $A_{1}$ is extreme and $C_{\infty}=C(A)=C\left(A_{1}\right)$, Theorem 1 yields the convergence

$$
d_{H}\left(n^{1 / \alpha} C_{n}\left(\pi_{1}\right), C_{\infty}\right) \rightarrow 0 \quad \text { a.s. as } n \rightarrow \infty .
$$

From (13) and (14), there exists $n_{1} \in \mathbb{N}$ such that, for all $n>n_{1}$,

$$
C_{\infty} \subset n^{1 / \alpha} C_{n}\left(\pi_{1}\right)^{(\epsilon)} \subset n^{1 / \alpha} C_{n}\left(\pi_{\alpha, v}\right)^{(\epsilon)} .
$$

It is easy to see that there exists $\tilde{v}_{i}, i \in I$, such that the set

$$
A_{2}=\left\{v_{j}^{1 / \alpha} e^{(j)}, j \in J ; \tilde{v}_{i}^{1 / \alpha} e^{(i)}, i \in\{1, \ldots, l\} \backslash J\right\}
$$

is extreme and satisfies the relation

$$
C_{\infty} \subset C\left(A_{2}\right) \subset C_{\infty}^{(\epsilon)} .
$$

From $\pi_{\alpha, \nu}$ we construct a second PPP $\pi_{2}$ by adding the independent point processes $\left(\tilde{\pi}_{i}\right)_{i \in J}$ such that $\left(\tilde{\pi}_{i}\right)_{i \in J}$ are independent of $\pi_{\alpha, v}$, and, for each $i \in J$, the spectral measure of $\tilde{\pi}_{i}$ is supported by $\mathcal{L}_{i}$ and the intensity measure is $\tilde{\mu}_{i}(A)=\left(\tilde{v}_{i}-v_{i}\right) \alpha \int_{A} r^{-\alpha-1} \mathrm{~d} r$.

According to Theorem 1 we have

$$
d_{H}\left(n^{1 / \alpha} C_{n}\left(\pi_{2}\right), C\left(A_{2}\right)\right) \rightarrow 0 \text { a.s., }
$$

and using Lemma 1 , we obtain, for all $n \in \mathbb{N}$,

$$
C_{n}\left(\pi_{\alpha, v}\right) \subset C_{n}\left(\pi_{2}\right) \text {. }
$$

From (16), (17), and (18), there exists $n_{2} \in \mathbb{N}$ such that, for all $n>n_{2}$,

$$
n^{1 / \alpha} C_{n}\left(\pi_{\alpha, v}\right) \subset n^{1 / \alpha} C_{n}\left(\pi_{2}\right) \subset C\left(A_{2}\right)^{(\epsilon)} \subset C_{\infty}^{(2 \epsilon)} .
$$

According to (15) and (19), for all $n \geq \max \left(n_{1}, n_{2}\right)$, we have

$$
n^{1 / \alpha} C_{n}\left(\pi_{\alpha, v}\right) \subset C_{\infty}^{(2 \epsilon)} \text { and } C_{\infty} \subset n^{1 / \alpha} C_{n}\left(\pi_{\alpha, v}\right)^{(2 \epsilon)}
$$

By the definition of $d_{H}$, this means that $d_{H}\left(n^{1 / \alpha} C_{n}\left(\pi_{\alpha, \nu}\right), C_{\infty}\right) \leq 2 \epsilon$, and we obtain (5). 


\section{Simulation and conjectures}

We now present the results of some simulations to investigate the limit shape and the asymptotic behavior of basic functionals in the case of continuous (namely uniform) spectral measures.

The point processes $\left\{x^{(j)}, j \in \mathbb{N}\right\}$ are simulated using representation (3). Let $C_{1, n}$ be the

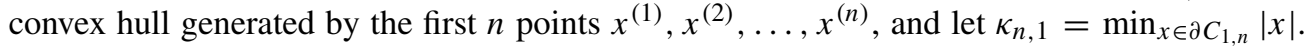
Since the points of the simulated PPP are ordered by their distances from the origin, it is evident that $C_{1, n^{\prime}}=C_{1}$ with $n^{\prime}=\min \left\{n: \kappa_{n, 1}>\left|x^{(n+1)}\right|\right\}$. This fact is used to construct the successive convex hulls $\left(C_{k}\right)_{k \in \mathbb{N}}$.

Figure 1 illustrates the behavior of the peels. The observed closeness of the peels to the unit circle also supports our conjecture that the limit shape exists, and is a circle.

It is of great interest to gain some insight into the possible behavior of the perimeter $\mathcal{L}$, the area $\mathcal{A}$, and the total number of vertices $\mathcal{N}$ of the convex polygons $C_{k}, k=1,2, \ldots$ It seems evident that $\mathcal{L}\left(C_{k}\right)$ and $\mathcal{A}\left(C_{k}\right)$ tend to 0 as $k \rightarrow \infty$. Intuitively, we expect that $\mathcal{N}\left(C_{k}\right) \rightarrow \infty$ as $k \rightarrow \infty$.

Figure 2(a)-(c) represent the logarithm of $\mathcal{L}\left(C_{k}\right), \mathcal{A}\left(C_{k}\right)$, and $\mathcal{N}\left(C_{k}\right)$ as functions of $\ln (k)$, calculated on a simulated PPP for different values of $\alpha$. The observed closeness of the points to straight lines makes it reasonable to expect that, in a sense,

$$
\mathcal{L}\left(C_{k}\right) \asymp k^{-\gamma_{l}}, \quad \mathcal{A}\left(C_{k}\right) \asymp k^{-\gamma_{a}}, \quad \mathcal{N}\left(C_{k}\right) \asymp k^{\gamma_{n}},
$$

with $\gamma_{l}$ and $\gamma_{a}$ depending on $\alpha$, whereas it seems that $\gamma_{n}$ does not dependent on $\alpha$.

The next step consists in estimating the exponents and possibly the dependence on $\alpha$. Using independent replications of PPPs, we estimate the three exponents defined in (20) for different values of $\alpha$. Figure 2(d) represents the logarithm of estimated exponents versus $\ln (\alpha)$. For the
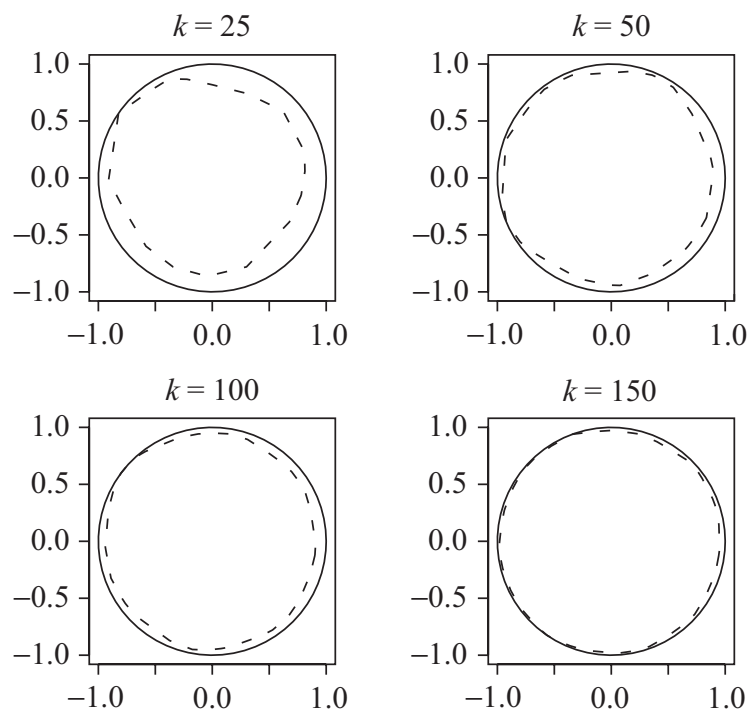

FIGURE 1: The normalized simulated shapes of $\hat{C}_{25}, \hat{C}_{50}, \hat{C}_{100}$, and $\hat{C}_{150}$ for the case in which $\alpha=\frac{3}{2}$ and the spectral measure is uniform (dotted lines). The unit circles are denoted by solid lines. 

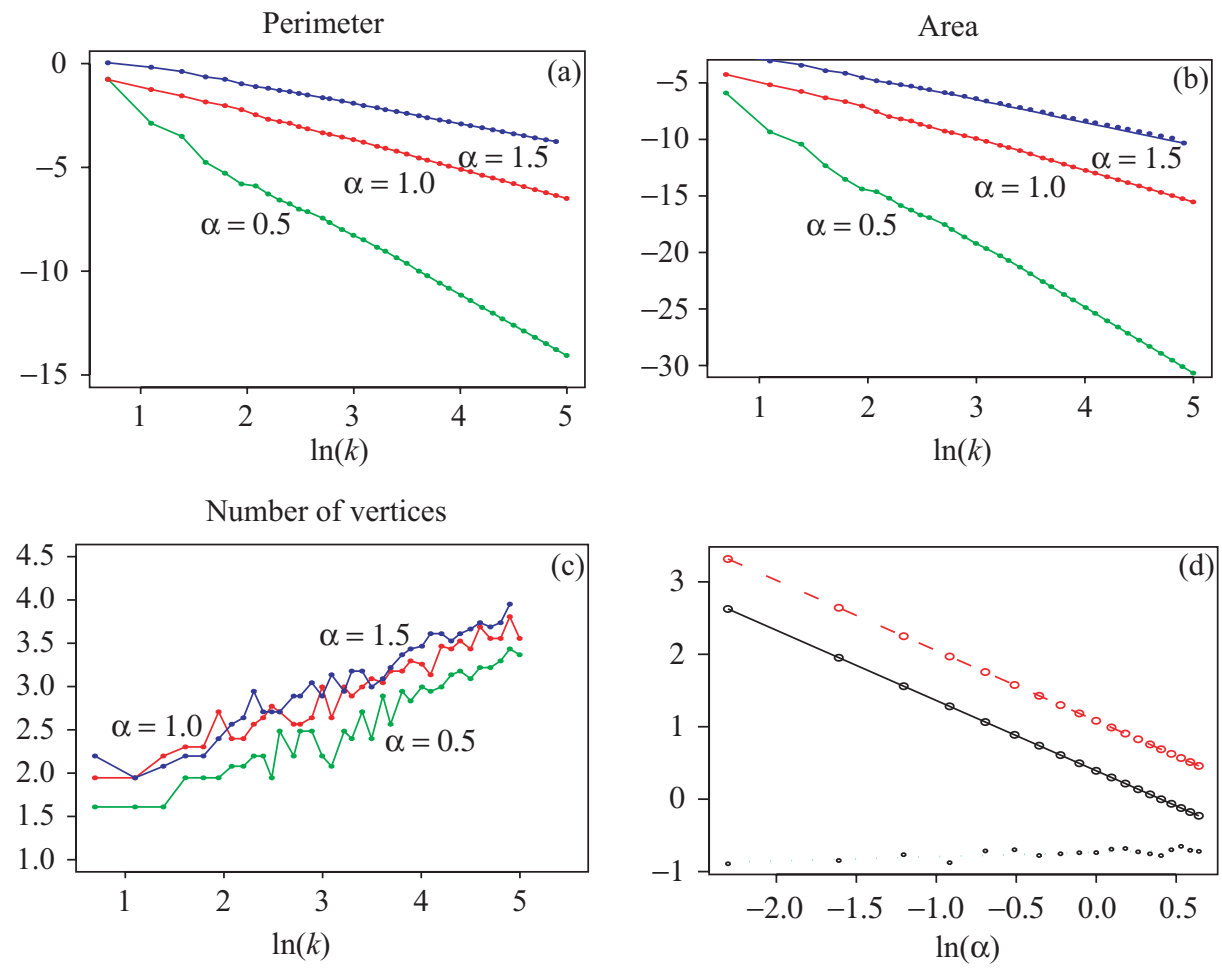

FIGURE 2: $\log -\log$ representations of the values of (a) $\mathcal{L}\left(C_{k}\right)$, (b) $\mathcal{A}\left(C_{k}\right)$, and (c) $\mathcal{N}\left(C_{k}\right)$ as functions of $k$. The functionals are calculated on a simulated PPP for different values of $\alpha, \alpha=0.5,1.0,1.5$, and the uniform spectral measure. (d) The estimated values of the logarithm of exponents defined in (20) versus $\ln (\alpha)$ and the best linear fittings $\ln \hat{\gamma}_{a}=-0.97 \ln \alpha+\ln 2.95, \ln \hat{\gamma}_{l}=-0.97 \ln \alpha+\ln 1.48$, and $\ln \hat{\gamma}_{n}=0.06 \ln \alpha+\ln 0.48$. Three exponents are estimated for each $\alpha$ on 1000 independent replications.

three cases, the linear approximation seems reasonable. According to the estimated coefficients of the straight lines (see the equations in the caption of Figure 2), it looks very credible that the true values are

$$
\gamma_{l}=\frac{3}{2 \alpha}, \quad \gamma_{a}=\frac{3}{\alpha}, \quad \text { and } \quad \gamma_{n}=\frac{1}{2} .
$$

The next plausible reasoning may confirm this conclusion. If we admit the value $\gamma_{n}=\frac{1}{2}$ then, after the $k$ th iterative step of the peeling procedure, the number of deleted points should be of the order of $\sum_{j=1}^{k} \mathcal{N}\left(C_{j}\right) \asymp k^{3 / 2}$ and

$$
\rho_{k} \asymp \max _{x \in C_{k}}|x| \asymp\left(k^{3 / 2}\right)^{-1 / \alpha} .
$$

By Remark 2, this convergence would lead to $\mathcal{L}\left(C_{k}\right) \asymp \rho_{k} \asymp k^{-3 /(2 \alpha)}$ and $\mathcal{A}\left(C_{k}\right) \asymp \rho_{k}^{2} \asymp$ $k^{-3 / \alpha}$, which is in agreement with the estimated values of $\alpha_{l}$ and $\alpha_{a}$ obtained in (21).

\section{Acknowledgements}

We are grateful to the anonymous referees for their suggestions, which have helped to improve the exposition of our paper. 


\section{References}

[1] Davydov, Yu. and Nagaev, A. V. (2004). On the role played by extreme summands when a sum of independent and identically distributed random vectors is asymptotically $\alpha$-stable. J. Appl. Prob. 41, 437-454.

[2] Davydov Yu., Molchanov, I. and Zuev, S. (2008). Strictly stable laws on convex cones. Electron. J. Prob. 13, 259-321.

[3] LePage, R., Woodroofe, M. and Zinn, J. (1981). Convergence to a stable distribution via order statistics. Ann. Prob. 9, 624-632.

[4] Resnick, S. I. (1987). Extreme Values, Regular Variation, and Point Processes. Springer, New York.

[5] Samorodnitsky, G. and Taqqu, M. S. (1994). Stable Non-Gaussian Random Processes. Chapman and Hall, New York. 